\title{
Efecto de la suplementación en vacas de pastoreo sobre la producción, eficiencia del uso y costo beneficio
}

\section{Effect of supplementation in grazing cows on production, efficiency of use and cost benefit ratio}

\author{
Francisco Adolfo Gutiérrez León ${ }^{1}$, Juan Rocha$^{1}$, Arnulfo Portilla ${ }^{1}$, Brayan Ruales ${ }^{1}$ \\ $凶$ Autor de correspondencia: fgutierrez@uce.edu.ec \\ 1 Universidad Central del Ecuador, Facultad de Ciencias Agrícolas, Ciudadela Universitaria, \\ Jerónimo Leiton s/n y Av. La Gasca, (593) 2052219, Quito- Ecuador.
}

\begin{abstract}
Resumen
Los pastos son el principal alimento para los rumiantes complementado con cereales. No obstante, los cereales son costosos en países donde no se producen excedentes, lo cual podría reducir la rentabilidad del negocio lechero. El objetivo de esta investigación fue determinar el efecto de la suplementación del balanceado en vacas de pastoreo, sobre producción de leche, la eficiencia del uso, la interacción con la biomasa del pasto y la relación costo beneficio. Se utilizó un diseño de cuadrado latino con cuatro animales y cuatro tratamientos. Los tratamientos a evaluar se determinaron a partir de la relación entre litros de leche y $\mathrm{kg}$ de concentrado. Las relaciones propuestas fueron de 2,5, $5,0,7,5$ y 10,0. Se determinó que la producción de leche (L/vaca/día) aumenta al incrementarse la ración. La interacción concentrado y biomasa $(\mathrm{kg} / \mathrm{MS})$ determinó que con una baja producción de biomasa en las pasturas $<1.564$ $\mathrm{kg}$ MS/ha se vuelve más evidente la necesidad de balanceado por parte de las vacas para mantener su producción. Si las pasturas tienen entre 2.330-1.564 kg MS/ha, las cantidades de balanceado a utilizar son más bajas; pero si las pasturas tienen $>2.330 \mathrm{~kg} \mathrm{MS} / \mathrm{ha}$, la cantidad de balanceado que debe recibir un animal pierde importancia. La eficiencia del uso del balanceado en la producción de leche tiene un comportamiento decreciente; esto es, a mayor cantidad de suplemento menor es la eficiencia en producción de leche. La relación costo beneficio es mejor cuando menor cantidad de suplemento se utiliza y decae cuando las cantidades de suplemento aumentan.
\end{abstract}

Palabras clave: pastos, lechería, rendimiento, cereales, balanceado.

\begin{abstract}
Pastures are the main feeding source for ruminants supplemented with cereals. However, cereals are expensive in countries where surpluses are not produced, which could reduce the profitability of the dairy business. The objective of this research was to determine the effect of supplementation in grazing cows, on milk production, the efficiency of use, interaction with grass biomass, and cost-benefit ratio. A Latin square design with four animals and four treatments were used. The treatments to be evaluated were determined from the ratio between liters of milk and $\mathrm{Kg}$. of concentrate. The proposed relationships were $2,5,5,0,7,5$, and 10,0. It was determined that milk production (L/cow/ day) increases when increasing the ration. The concentrated interaction and biomass ( $\mathrm{kg} / \mathrm{MS})$ determined that with low production of biomass in pastures under $<1.564 \mathrm{~kg} \mathrm{DM} /$ hect. it becomes more evident the need for balance by the cows to maintain their production. If pastures have between $2.330-1.564 \mathrm{~kg} \mathrm{DM} /$ hect. the balancing amounts to be used are lower; but if pastures have $>2.330 \mathrm{~kg} \mathrm{DM} /$ hect. the amount of balance that an animal should receive loses significance. The efficiency of the use of the balance in milk production has a decreasing behavior, that is, the greater the amount of supplement, the lower efficiency in milk production. The cost-benefit ratio is better when less supplement is used and decreases when the supplement amounts increase.
\end{abstract}

Keywords: grass, dairy, performance, cereals, balanced. 


\section{Introducción}

El uso de pasturas en vacas lecheras resulta en un sistema de alimentación de bajo costo ya que las pasturas son la fuente de nutrientes más barata (Delaby, Peyraud \& Delagarde, 2001). Un sistema de pastoreo intensivo, consiste en que la alimentación del ganado durante los 365 días del año es por pastoreo más suplementación estratégica que complementa al pasto (Alonso, 2016). Los sistemas pastoriles eficientes se caracterizan por una alta producción de leche por unidad de superficie, mientras que los sistemas en confinamiento se caracterizan por una alta producción por vaca (Clark \& Kanneganti, 1998).

El uso de piensos concentrados para rumiantes se limita a los países donde los precios de la carne y la leche son relativamente más elevados que los de cereales. Sin embargo, en lugares donde los precios de los cereales son más altos que los de la carne y leche, situación típica en los países en desarrollo, la alimentación de rumiantes con cereales forrajeros no resulta rentable (Steinfeld et al., 2009).

Los costos de alimentación en vacunos representa entre el $45-60 \%$ del costo total de producción de leche (Heinrichs, 2015) (Gutiérrez, 2015), siendo el pasto la fuente más rentable. Según Rua (2016) la producción de leche con pastos es hasta 10 veces menor que la basada en concentrados. Aunque la producción de leche con base en pastos produce menos leche en relación a un sistema estabulado, el productor debe enfocarse en que el margen entre egresos e ingresos sea más amplio. Salado (2011) estableció que las ganaderías a nivel mundial son más eficientes cuando mayor cantidad se pasto incluyen en la alimentación, como es el caso de Nueva Zelanda y Australia.

Para Batallas (2015) la lechería ecuatoriana se desarrolla en un sistema productivo donde predomina el pastoreo de forrajes de variable calidad. Esta práctica (el pastoreo) es sin lugar a dudas la más lógica y recomendable en un país donde tenemos condiciones favorables para realizarla; más aún si consideramos que la estructura de costos de producción y el precio actual de la leche inevitablemente obligan al productor a implementar prácticas alimenticias de bajo costo y alta rentabilidad.

El propósito de esta investigación fue determinar el efecto de diferentes raciones de balanceado en vacas de pastoreo, y evaluar su respuesta en la producción de leche; cómo interactúa la producción de pasturas con la ración de balanceado; analizar el comportamiento de la eficiencia del uso del balanceado para la producción de leche y determinar cuál es la mejor relación costo beneficio.

\section{Materiales y Métodos}

El estudio se realizó en el Campus Académico Docente Experimental "La Tola" de la Facultad de Ciencias Agrícolas de la Universidad Central del Ecuador, ubicada en la parroquia de Tumbaco del cantón Quito, provincia de Pichincha, a 2.465 m.s.n.m., latitud $00^{\circ}$ $14^{\prime} 46^{\circ}$ 'S, longitud $78^{\circ} 22^{\prime} 00^{\prime}$ ' O, temperatura máxima: $22,7{ }^{\circ} \mathrm{C}$, mínima: $12,3{ }^{\circ} \mathrm{C}$, promedio: 17,6 ; precipitación anual $840 \mathrm{~mm}$ y humedad relativa del $78 \%$.

Se seleccionaron 4 vacas de raza Holstein-Friesian, cada una de las cuales se encontraba entre 100 y 115 días de lactancia, con más de un parto, peso promedio $550 \mathrm{~kg}$, edad entre 3 y 5 años y en condiciones óptimas de salud. Cada vaca fue una unidad experimental. Se evaluaron cuatro tratamientos que surgen de la relación entre la producción de leche (L/ vaca/día) y la cantidad de concentrado ( $\mathrm{kg} /$ día), la idea fue determinar diferentes dosis de concentrado de acuerdo a la producción de leche (véase Tabla 1). Se utilizó un balanceado comercial peletizado, con el $14 \%$ de proteína bruta (PB), extracto etéreo (EE) $5 \%$, fibra bruta (FB) $17 \%$, cenizas $10 \%$, y extracto no nitrogenado (ENN) 54\%.

Tabla 1. Tratamientos establecidos para la evaluación del "Efecto de la relación entre la cantidad de balanceado y producción de leche en vacas de segundo tercio de lactancia"

\begin{tabular}{ccc}
\hline No. de Tratamiento & Tratamientos & $\begin{array}{c}\text { Relación entre litros de leche/vaca/día y } \\
\mathbf{1} \text { kg de balanceado }\end{array}$ \\
\hline 1 & $\mathrm{~T}_{1}$ & 2,5 \\
2 & $\mathrm{~T}_{2}$ & 5,0 \\
3 & $\mathrm{~T}_{3}$ & 7,5 \\
4 & $\mathrm{~T}_{4}$ & 10,0 \\
\hline
\end{tabular}


Se utilizó un diseño experimental cuadrado latino, usando cuatro unidades experimentales con cuatro tratamientos. Este diseño permite que los tratamientos sean evaluados en todas las unidades experimentales en función del tiempo.
Para determinar diferencias estadísticas entre tratamientos se utilizó la prueba de Tukey al 5\%; el programa estadístico utilizado fue el InfoStat (véase Tabla 2), el diseño estadístico tiene el siguiente modelo matemático.

$$
Y_{i j k}=\mu+B_{i}+A_{j}+P_{K}+\text { interaccion }+E_{i j k}
$$

\begin{tabular}{|l|l|}
\hline$Y_{i j k}$ & \multicolumn{1}{|c|}{ Producción de leche } \\
\hline \multirow{3}{*}{$B_{i}$} & $\mathrm{i}=1: 2,5 \mathrm{l} /$ vaca/día: $1 \mathrm{~kg}$ de balanceado \\
& $\mathrm{i}=2: 5,01 /$ vaca/día: $1 \mathrm{~kg}$ de balanceado \\
$\mathrm{i}=3: 7,51 /$ vaca/día: $1 \mathrm{~kg}$ de balanceado \\
$\mathrm{i}=4: 10,01 /$ vaca/día: $1 \mathrm{~kg}$ de balanceado
\end{tabular}

Cada tratamiento fue evaluado por dos semanas y hubo una etapa de transición para cambiar de tratamiento que fue de 7 días, en los cuales se cambió de tratamiento de manera progresiva a los animales.
Las variables para evaluar fueron: producción de leche (L/vacas/día), la eficiencia del uso del balanceado, la interacción materia seca en los pastos y balanceado y relación costo beneficio.

Tabla 2. Distribución de los tratamientos para la evaluación del "Efecto de la relación entre la cantidad de balanceado y producción de leche en vacas de segundo tercio de lactancia"

\begin{tabular}{ccccc}
\hline Animal & \multicolumn{3}{c}{ Tratamientos } \\
\hline Animal 1 & $\mathrm{T} 1$ & $\mathrm{~T} 2$ & $\mathrm{~T} 3$ & $\mathrm{~T} 4$ \\
Animal 2 & $\mathrm{T} 4$ & $\mathrm{~T} 1$ & $\mathrm{~T} 2$ & $\mathrm{~T} 3$ \\
Animal 3 & $\mathrm{T} 3$ & $\mathrm{~T} 4$ & $\mathrm{~T} 1$ & $\mathrm{~T} 2$ \\
Animal 4 & $\mathrm{T} 2$ & $\mathrm{~T} 3$ & $\mathrm{~T} 4$ & $\mathrm{~T} 1$ \\
\hline
\end{tabular}

La producción de leche (L/vaca/día), se acopló el sistema de ordeño a bidones de $40 \mathrm{~L}$, y se recolectó la producción de cada vaca de manera independiente, en un decalitro se determinó el volumen (L). Este proceso se realizó en los ordeños de la mañana y la tarde, y se sumó los dos valores para determinar la producción total diaria.

La producción de biomasa ( $\mathrm{kg} \mathrm{MS} / \mathrm{ha}$ ) se determinó antes del pastoreo de cada potrero, el intervalo entre pastoreos fue entre 28 y 30 días. Fisiológicamente el pasto en las condiciones agroclimáticas del experimento llega a su punto máximo de crecimiento y es antes de que empiece su senescencia; la investigación se desarrolló en la época de invierno entre los meses de enero y abril. El método utilizado fue el del cuadrante, con las siguientes dimensiones $0,5 \times 0,5 \mathrm{~m}$, se realizó un muestreo al azar y se tomaron 10 muestras, en cada una de ellas se realizó un corte a ras de suelo y se pesó la biomasa acumulada, una muestra de la biomasa se secó en una estufa por 24 horas a $70^{\circ} \mathrm{C}$ (Mónaco, Santa, Rosa \& Autran, 2017), finalmente se determinó el contenido de MS y se calculó la producción total por hectárea. 
La eficiencia de uso del balanceado se determinó utilizando la ley de rendimientos decrecientes (también llamada rendimientos marginales decrecientes): es la disminución marginal del incremento del producto o servicio (por unidad) en un proceso productivo confor- me se añaden nuevos factores productivo (Hernández, 2017). Se relacionó la producción (L/vaca/día) con la cantidad de balanceado ( $\mathrm{kg} / \mathrm{vaca} /$ día) y se estimó la eficiencia de uso para cada uno de los tratamientos, aplicados de acuerdo con la siguiente fórmula:

$$
\text { Eficiencia de uso }=\frac{P L}{B}
$$

Donde PL es la producción de litros de leche vaca/día en cada uno de los tratamientos y B es la cantidad de balanceado suministrado para obtener el PL en cada tratamiento.

El análisis de costo-beneficio, se determinó con el método análisis marginal de costos beneficios (Cohen, 2016). Se tomaron en cuenta los ingresos por venta de leche y los egresos por costos de alimentación como balanceado, estos se relacionaron para estimar cuál es el ingreso en dólares por leche por cada dólar invertido en balanceado.

\section{Resultados y Discusión}

\subsection{Producción de leche}

La producción de leche aumentó conforme se incrementó la cantidad de concentrado suministrado a las vacas como complemento al pastoreo. El T1 tuvo la mayor respuesta en producción de leche, en promedio las vacas consumieron $8 \mathrm{~kg}$ de concentrado, mientras que en el T2, T3 y T4 tuvieron una menor producción y consumieron en promedio $1,7 \mathrm{~kg}$ de concentrado. La mejor respuesta en el T1 se debió a que las vacas tuvieron una mayor disponibilidad de MS oferta de pasto en el potrero y concentrado en el ordeño, y como resultado un más alto consumo de energía que se tradujo en una mayor producción de leche. Por otro lado, el T4 obtuvo una menor disponibilidad de MS, si bien tuvo la misma oferta de pasto en el potrero, la cantidad de concentrado ofertada en el ordeño disminuyó, por lo que su consumo de energía fue menor y su producción de leche fue inferior.

El bajo consumo de MS y energía metabolizable y la falta de sincronía en el rumen entre la energía y la proteína cruda aportada por el forraje, son los principales factores que limitan la producción de leche en pastoreo (Stockdale, 2000). Por tanto, la inclusión de alimentos suplementarios, en forma de concentrados, son necesarios para incrementar el consumo de energía y de materia seca total (McGilloway \& Mayne, 1996). Una vaca en pastoreo exclusivamente, es capaz de consumir suficiente energía como para producir alrededor de 26 litros de leche al día con pérdida mínima de su condición corporal, esto con pasturas con $15 \% \mathrm{MS}, 27 \% \mathrm{PC}$, $2,85 \mathrm{Mcal} / \mathrm{kg}$ energía metabolizable y $36,5 \% \mathrm{FDN}$. Sin embargo, el periodo de óptima composición nutricional y disponibilidad de praderas solo son posibles en determinadas épocas del año, como es la primavera (Klein, 2003). Si no se utilizan forrajes de alta calidad, se hace necesario altos niveles de suplementación a fin de obtener los rendimientos por vaca requeridos o establecidos como meta (Albarrán Portillo, 1999).

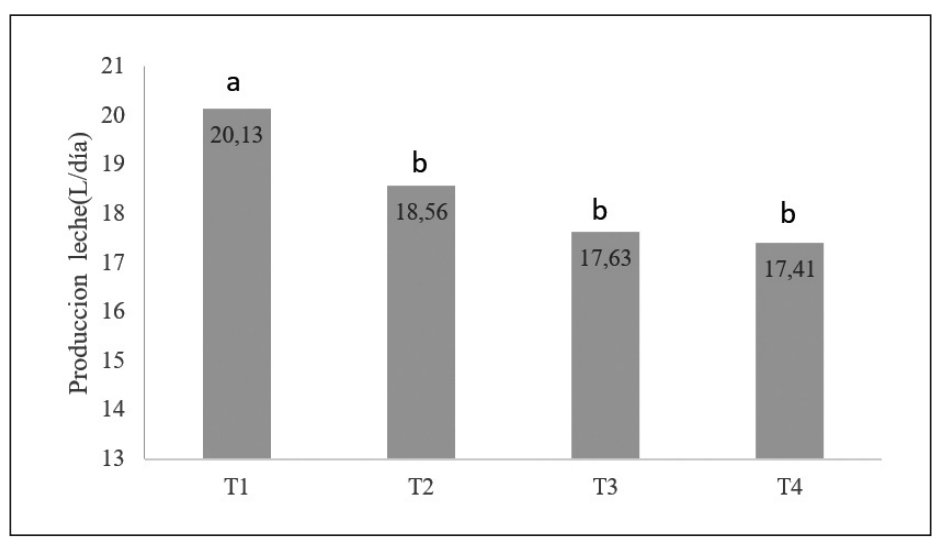

Figura 1. Influencia de la cantidad de balanceado sobre la producción de leche/vaca/día. 


\subsection{Eficiencia del uso de balanceado}

Para vacas lecheras de segundo tercio de lactancia, la cantidad de concentrado recomendable en esta fase es $2,4 \mathrm{~kg}$ concentrado/vaca/día con una producción de 18,6 L/vaca/día y una eficiencia de uso de $6,5 \mathrm{~kg}$ de leche por cada $\mathrm{kg}$ de concentrado (véase Figura 4 y Tabla 3).

Vélez (2015) establece que para vacas con alto mérito genético, la respuesta a la suplementación puede llegar a los $19 \mathrm{~kg}$ leche/vaca/día con una ingesta de $2,7 \mathrm{~kg}$ de concentrado, mientras que las vacas de mediana producción alcanzan valores de 17 $\mathrm{kg}$ leche/vaca/día con la misma ración de concentrado. Bretschneider \& Salado (2010) concluyeron que el umbral de suplementación que maximiza la producción de leche sin afectar la salud del rodeo es de $7 \mathrm{~kg}$ de balanceado. Debido a que por encima de un determinado nivel de suplementación la respuesta productiva por cada unidad adicional de suplemento es menor, niveles muy altos de suplementación podrían no resultar en una mayor rentabilidad.

Trabajos realizados por Guevara, Guevara \& Serpa (2017) en relación con el genotipo concluyen que las vacas del tipo neozelandés, fueron más eficientes en la producción de leche con $16,2 \mathrm{~kg} / \mathrm{a} / \mathrm{d}$ en comparación con las del tipo norteamericano que registraron una producción de $12,5 \mathrm{~kg} / \mathrm{a} / \mathrm{d}$. Por otro lado, Gaona et al. (2015) sostienen que, si bien la genética es importante, la mayor limitante para la evolución en la producción de los animales es la nutrición y alimentación de los animales.

La mejor respuesta en pastoreo se obtiene con suministros inferiores a 5-6 kg/vaca. Sobre estos niveles, la respuesta al concentrado disminuye a menos que la oferta de pradera por vaca se restrinja (Anrique \& Vásquez, 2013).

Tabla 3. Eficiencia de uso del balanceado a diferentes cantidades

\begin{tabular}{cccc}
\hline Tratamientos & $\begin{array}{c}\text { Balanceado (B) } \\
\mathrm{kg} / \text { vaca/día }\end{array}$ & $\begin{array}{c}\text { Producción Leche } \\
\text { (PL) L/vaca/día }\end{array}$ & $\begin{array}{c}\text { Eficiencia de uso kg de } \\
\text { leche/kg de concentrado }\end{array}$ \\
\hline T4 & 1,7 & 17,4 & 10 \\
T3 & 2,4 & 17,6 & 7,5 \\
T2 & 3,7 & 18,6 & 5 \\
T1 & 8,1 & 20,3 & 2,5 \\
\hline
\end{tabular}

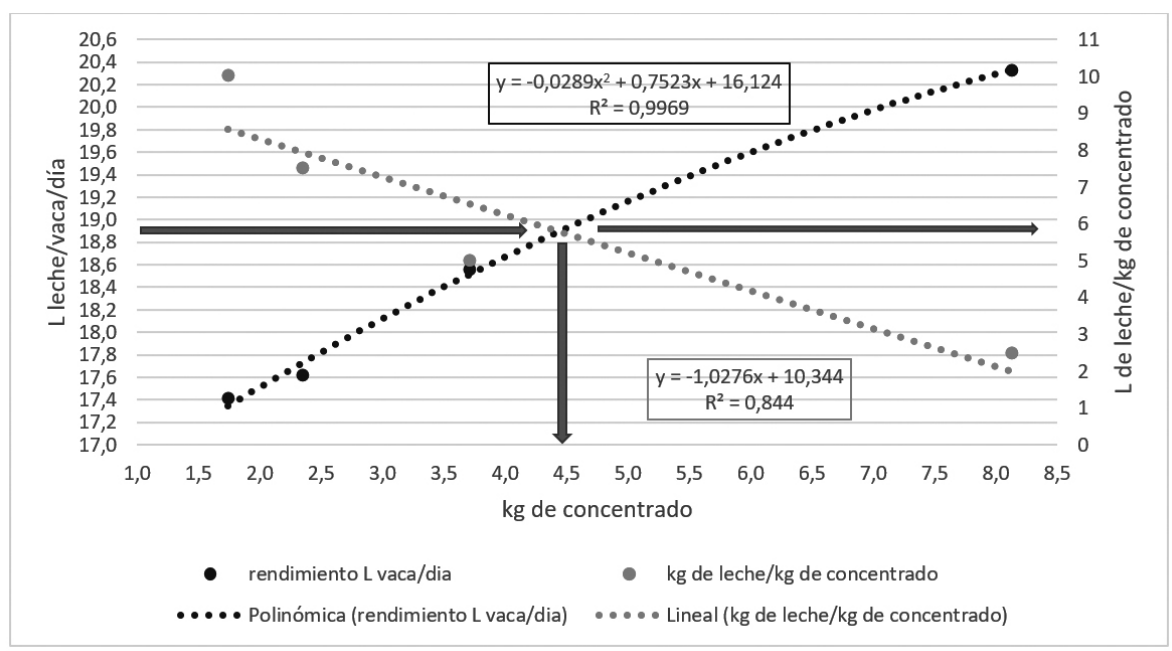

Figura 2. Eficiencia del uso de balanceado y efecto sobre la producción de leche en vacas de segundo tercio de lactancia. 


\subsection{Interacción MS del pasto y cantidad de balanceado}

Las producciones de MS de los potreros interactuaron de diferente manera con los tratamientos suministrados a los animales (Figura 3). Cuando las pasturas tienen un bajo rendimiento $<1.564 \mathrm{~kg} \mathrm{MS} / \mathrm{ha}$ los mejores resultados se tienen con altos niveles de balanceado como es el T1, seguido del T4, T2 y T3. Si la producción de las pasturas está en un nivel intermedio entre 1.564 - $2.330 \mathrm{~kg} \mathrm{MS} / \mathrm{ha}$ la mayor producción se alcanzó con el T1, seguido del T2, T3 y T4. Finalmente, cuando las pasturas tienen altos rendimientos $>2.330 \mathrm{~kg} \mathrm{MS} / \mathrm{ha}$ los tratamientos no presentaron diferencia estadística, es decir que los animales ya no responden a altas cantidades de concentrado y pueden mantener su producción incluso con cantidades bajas de balanceado.

Bargo (2012) afirma que la tasa de sustitución aumenta y la respuesta a la suplementación baja a medida que la disponibilidad de pastura aumenta. Grainger \& Mathews (1989) demuestran en su estudio que la tasa de sustitución de la pastura en relación al concentrado aumentó y la respuesta a la suplementación disminuyó, a medida que se aumentó la disponibilidad de forraje. Ferreira, Schneider \& Clavijo Villamizar (2015), mencionan que la sustitución de la pastura se da por su calidad; pasturas con una buena calidad tienen una menor sustitución por el balanceado.

Para Carulla, Cárdenas, Sánchez \& Riveros (2004) el consumo es uno de los factores más importantes para determinar la producción animal. Se estima que el $70 \%$ de las variaciones en la producción animal en pastoreo se pueden explicar por la variación en el consumo de alimento. Auldist, Thomson, Mackle, Hill $\&$ Prosser (2000) reportan que un incremento en la oferta de forraje aumenta la producción de leche. Se ha propuesto también que al incrementar la oferta de forraje se presenta una mayor selectividad por parte del animal mejorando el balance energético (Wales, Doyle \& Dellow, 1998). Riquelme \& Pulido (2008) concluyeron que distintos niveles de suplementación con concentrado, utilizando praderas de elevada calidad nutritiva y con gran disponibilidad de forraje, disminuyen el consumo de materia seca de la pradera, sin incrementar el consumo total de materia seca.

Es aceptado que la respuesta a la suplementación en este tipo de ensayos depende de la tasa de sustitución, de la calidad del suplemento en relación con la calidad de la pradera, y de la partición de la energía adicional consumida entre producción de leche y ganancia de peso corporal (Bargo, Muller, Kolver \& Delahoy, 2003). Pulido, Cerda \& Stehr (1999), mencionan que por cada $\mathrm{kg}$ de alimento balanceado consumido en promedio se sustituye el consumo de 0,432 y $1,064 \mathrm{~kg}$ MS de forraje pastoreando.

La respuesta en volumen de leche al incremento en la oferta forrajera varía dependiendo del tercio de lactancia. En el primer tercio la respuesta es lineal mientras que en los otros tercios ya no se observa una respuesta en volumen de producción, pero sí en el contenido de grasa y proteína (Mojica et al., 2009).

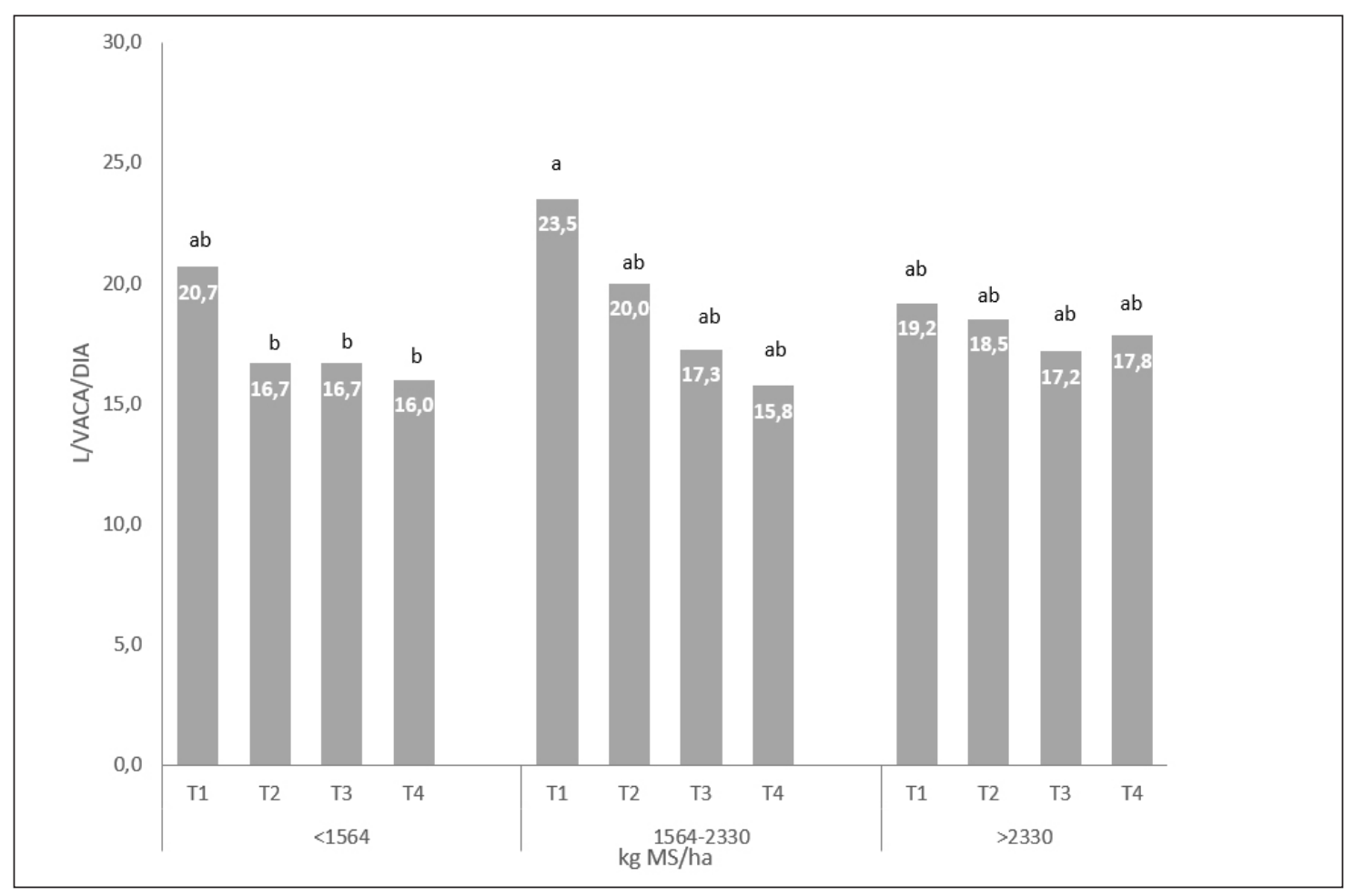

Figura 3. Interacciones entre producción de MS del potrero y las diferentes raciones de balanceado con la producción de leche. 


\subsection{Relación costo beneficio}

El tratamiento que mejor relación costo beneficio tuvo fue el T4 en el cual el balanceado representa solo el 9\% del valor de un litro de leche, conforme aumenta las cantidad de balanceado, como es el caso del T3 y T2, aumenta también la relación al 13\% y 19\%. Finalmente, el T1 tuvo una inferior relación costo beneficio, el balanceado representó el $38 \%$ del valor del costo de un litro de leche (Tabla 4). Al aumentar la ración de concentrado $(\mathrm{kg} / \mathrm{vaca} /$ día) la relación costo beneficio disminuyó. Esto obedece a que la producción de leche no aumentó en la proporción necesaria para mejorar la relación costo beneficio. Por otro lado, el costo de cada $\mathrm{kg}$ de balanceado es de 0,45 dólares y el valor que se obtiene por cada litro es de 0,48 dólares, es decir que la relacion entre los dos es cercana a uno. Por lo tanto, para que se obtenga un rédito económico por cada $\mathrm{kg}$ de balanceado suministrado se debería producir más de un litro de leche adicional.

Los forrajes pueden contribuir a incrementar la eficiencia productiva de las explotaciones pecuarias, pues constituyen la fuente más barata, por lo que antes de recurrir al uso de concentrados, deben asegurarse forrajes de buena calidad (Hodgson, 1994). El aporte de suplementos debe realizarse teniendo presente que la pradera es el alimento más económico y debe participar lo más posible en la ración. El uso de suplementos permite reforzar el consumo así como la calidad de la ración y la producción de leche (Anrique \& Vásquez, 2013).
Salado, Maciel, Bretschneider, Cuatrin \& Castignani (2014) mencionan que a pesar de los mayores ingresos del sistema confinado con respecto al sistema pastoril con suplementación, los litros libres de alimentación resultaron inferiores, debido a los mayores costos de la ración. El sistema pastoril con suplementación produjo menos leche, tuvo menores costos de alimentación, más litros libre de alimentación y similar comportamiento reproductivo comparado con un grupo confinado (Ghiano et al., 2016). Si bien la producción de leche obtenida bajo pastoreo y con suplementación energética es significativamente menor que la producción de vacas confinadas y alimentadas con una racion total mixta (TMR) existen variables que hacen al sistema pastoril económicamente competitivo. Estas variables incluyen el menor costo de la dieta base pastura (31\%), la menor incidencia de mastitis y la reducción en la mano de obra e instalaciones requeridas. Es decir, los menores costos de producción asociados al pastoreo son más que suficientes para compensar la menor producción de leche y mantener o mejorar la rentabilidad con respecto al sistema confinado. Bretschneider \& Salado (2010) demostraron que en escenarios de bajo precio de la leche y de alto costo del alimento, incrementos en la producción de leche de hasta un $36 \%$ en vacas de alta producción (45 L/día) confinadas y alimentadas con TMRs, no fueron suficientes para revertir la mejor rentabilidad a favor del sistema pastoril.

Tabla 4. Análisis de costos beneficio marginal de los tratamientos

\begin{tabular}{|c|c|c|c|c|}
\hline Tratamientos Variables & $\mathrm{T} 1$ & $\mathrm{~T} 2$ & $\mathrm{~T} 3$ & $\mathrm{~T} 4$ \\
\hline Producción L/vaca/día & 20,1 & 18,6 & 17,6 & 17,4 \\
\hline Relación L leche/ kg concentrado & 2,5 & 5 & 7,5 & 10 \\
\hline Ración kg de concentrado/vaca/día & 8,1 & 3,7 & 2,4 & 1,7 \\
\hline Valor de concentrado USD (saco de $40 \mathrm{~kg}$ ) & 18 & 18 & 18 & 18 \\
\hline Presentación kg & 40 & 40 & 40 & 40 \\
\hline valor USD $/ \mathrm{kg}$ & 0,45 & 0,45 & 0,45 & 0,45 \\
\hline Costo de ración día (USD) & 3,6 & 1,7 & 1,1 & 0,8 \\
\hline Valor USD/ litro de leche & 0,48 & 0,48 & 0,48 & 0,48 \\
\hline Ingreso por venta de leche en USD & 9,7 & 8,9 & 8,5 & 8,4 \\
\hline$\%$ del costo de balanceado y en cada $L$ de leche & $38 \%$ & $19 \%$ & $13 \%$ & $9 \%$ \\
\hline
\end{tabular}




\section{Conclusiones}

La cantidad de balanceado tiene una relación directamente proporcional con la producción de leche, al aumentar la ración diaria de balanceado por animal se incrementa la producción de L/vaca/ día. Sin embargo, la eficiencia de uso del balanceado disminuye al aumentar la ración de balanceado, la mejor eficiencia de uso se alcanza con $4,5 \mathrm{~kg}$ de concentrado con una producción de leche de 18,8 litros y la mejor relación es de $5,5 \mathrm{~kg}$ de concentrado/ producción de leche. La cantidad

\section{Referencias}

Albarrán Portillo, B. (1999). Evaluación del pastoreo de praderas irrigadas en las estrategias de alimentación de sistemas de producción de leche en pequeña escala. Universidad Autónoma del Estado de México, México. Toluca

Alonso, E. (2016). Viabilidad técnica y financiera del cambio del sistema convencional al pastoreo rotacional intensivo del hato lechero de la hacienda Santa Elisa, Danlí. Escuela Agrícola Panamericana, Zamorano, Honduras.

Anrique, R., \& Vásquez, J. (2013). Nutrición y alimentación de vacas lecheras en pastoreo Vol. 2. C. Bizama (Ed.) (pp. 1-66). Chile: CORFO

Auldist, M., Thomson, N., Mackle, T., Hill, J., \& Prosser, C. (2000). Effects of pasture allowance on the yield and composition of milk from cows of different $\beta$-lactoglobulin phenotypes. Journal of dairy science, 83(9), 2069-2074. https:// doi.org/10.3168/jds.S0022-0302(00)75088-0

Bargo, F. (2003). Suplementación en pastoreo: conclusiones sobre las últimas experiencias en el mundo. Facultad de Agronomía de la Universidad de Buenos Aires, 1. Obtenido de: https:// www.agro.uba.ar/sites/default/files/catedras/ bargo.pdf

Bretschneider, G., \& Salado, E. (2010). Sistemas confinados vs. pastoriles. Ventajas y desventajas. Ficha Técnica $\mathrm{N}^{\circ} 8$. Argentina: INTA-Proyecto Lechero.

Carulla, J., Cárdenas, E., Sánchez, N., \& Riveros, C. (2004). Valor nutricional de los forrajes más y calidad de la pastura en pastoreo es el factor que más limita la respuesta del balanceado, cuando existe una baja producción de pasturas los animales son dependientes de la cantidad de balanceado suministrado, pero si existe una mayor producción de pasturas la cantidad de leche que produce una vaca al día se mantiene independientemente de la cantidad de balanceado suministrado. La relación costos beneficio declina al aumentar la ración de balanceado, esto se debe a que la producción no aumenta en la cantidad necesaria para disminuir los costos del balanceado.

usados en los sistemas de producción lechera especializada de la zona andina colombiana. En: Seminario Nacional de Lechería Especializada: 'Bases Nutricionales y su Impacto en la Productividad". Eventos y Asesorías Agropecuarias EU (ed). Medellín, Septiembre 1 y 2. p.21-38.

Clark, D., \& Kanneganti, V. (1998). Grazing management systems for dairy cattle. En Grass for Dairy Cattle, Cherney, J.H., \& Cherney, D.J.R. (eds), (311-334). Wallingford, , UK: CABI Publishing.

Delaby, L., Peyraud, J., \& Delagarde, R. (2001). Effect of the level of concentrate supplementation, herbage allowance and milk yield at turn-out on the performance of dairy cows in mid lactation at grazing. Animal Science, 73(1), 171-181. https://doi.org/10.1017/ S1357729800058161

Ferreira, M., Schneider, R., \& Clavijo Villamizar, E. (2015). Efecto de dos disponibilidades de pradera sobre la tasa de sustitución al ofrecer alimentos balanceados en vacas lecheras. Universidad de la Salle, La Calera-Colombia.

Enciclopedia Financiera. (2018). Ley de rendimientos decrecientes. Obtenido de: https://www. enciclopediafinanciera.com/definicion-rendimientos-decrecientes.html

Gaona, R., Alegría, K., Terranova, M., Hernández, E., Benavides, R., Guerrero, H., . . . Patiño, L. (2015). El mejoramiento genético y la producción de leche. La esencia de una reali- 
dad de producción animal. Acta Agronómica, 64(3sup), 296-306. https://doi.org/10.15446/ acag.v64n3sup.50263

Ghiano, J., Costamagna, D., Taverna, M., Walter, E., Leva, P., Toffoli, G., . . Idone, L. (2016). Sistema de ordeño voluntario en vacas lecheras en pastoreo con dieta parcialmente mezclada en la cuenca lechera santafesina durante el período estival. Información técnica de produccción anima 2016l, 4(2), 22-33.

Grainger, C., \& Mathews, G. (1989). Positive relation between substitution rate and pasture allowance for cows receiving concentrates. Australian Journal of Experimental Agriculture, 29(3), 355-360. https://doi.org/10.1071/EA9890355

Guevara, R., Guevara, G., \& Serpa, G. (2017). Respuesta productiva de vacas lecheras neozelandesas sometidas a pastoreo rotacional en el trópico alto del norte de Ecuador. Maskana, 8(22), 153-155.

Hodgson, J. (1994). Manejo de pastos: teoría y práctica (Vol. 1). México.

Klein, F. (2003). Utilización de praderas y nutrición de vacas a pastoreo. En: Seminario: Hagamos de la lechería un mejor negocio. Instituto de Investigaciones Agropecuarias. Centro Regional de Investigación Remehue. Serie Actas, (24), 1-13.

Martínez, J. (2014). Análisis de costo beneficio. Ejemplos de análisis sector privado. Obtenido de: http://gis.jp.pr.gov/Externo_Econ/Talleres/ PresentationCB_JP_ETI.pdf

McGilloway, D., \& Mayne, C. (1996). Importance of grass availability for the high genetic merit dairy cow. Irelanda: Agricultural Research Institute of Northern Ireland.

Mojica, J., Castro, E., León, J., Cárdenas, E., Pabón, M., \& Carulla, J. (2009). Efecto de la oferta de pasto kikuyo (Pennisetum clandestinum) sobre la producción y calidad composicional de la leche bovina. Livestock Research for Rural Development, 21(1), 1-12.

Mónaco, N., Santa, V., Rosa, M., \& Autran, V. (2017). Evaluación de métodos indirectos para estimar biomasa en un pastizal natural del sur de Córdoba (Argentina Central). European
Scientific Journal, ESJ, 13(36), 59-70. http:// dx.doi.org/10.19044/esj.2017.v13n36p59

Pulido, R., Cerda, M., \& Stehr, W. (1999). Efecto del nivel y tipo de concentrado sobre el comportamiento productivo de vacas lecheras en pastoreo primaveral. Archivos de medicina veterinaria, 31(2), 177-187. http://dx.doi.org/10.4067/ S0301-732X1999000200004

Riquelme, C., \& Pulido, R. (2008). Efecto del nivel de suplementación con concentrado sobre el consumo voluntario y comportamiento ingestivo en vacas lecheras a pastoreo primaveral. Archivos de medicina veterinaria, 40(3), 243-249. http://dx. doi.org/10.4067/S0301-732X2008000300004

Salado, E., Maciel, N., Bretschneider, G., Cuatrin, A., \& Castignani, H. (2014). Sistemas de alimentación combinando pastura y ración totalmente mezclada: respuesta productiva y comportamiento reproductivo de vacas lecheras. Obtenido de: https://inta.gob.ar/sites/default/files/script-tmpinta_sistema_alimentacion_combinando_pastura_y_tmr_re.pdf

Steinfeld, H., Gerber, P., Wassenaar, T., Castel, V., Rosales, M., \& De Haan, C. (2009). La larga sombra del ganado: problemas ambientales $y$ opciones Vol. 1. Roma: FAO.

Stockdale, C. (2000). Levels of pasture substitution when concentrates are fed to grazing dairy cows in northern Victoria. Australian Journal of Experimental Agriculture, 40(7), 913-921. https://doi.org/10.1071/EA00034

Wales, W., Doyle, P., \& Dellow, D. (1998). Dry matter intake and nutrient selection by lactating cows grazing irrigated pastures at different pasture allowances in summer and autumn. Australian Journal of Experimental Agriculture, 38(5), 451-460. https://doi.org/10.1071/EA98043 